\title{
DMP in the putative promoter region of TLR9 in the superior temporal gyrus postmortem brain tissue in patients with Alzheimer's disease.
}

\author{
Yu Sun ${ }^{1,2}$ and Qingqin $S \mathrm{Li}^{1,2}$ \\ ${ }^{1}$ Neuroscience Therapeutic Area, Janssen Research \& Development, LLC, 1800 American Blvd, Pennington, NJ 08534 \\ ${ }^{2}$ Research Information Technology, Janssen Research \& Development, LLC, 1800 American Blvd, Pennington, NJ 08534
}

\begin{abstract}
Innate immune response mediated by Toll-like receptors (TLRs) has been implicated to be the therapeutic targets for Alzheimer's disease (AD). TLR9 agonist cytosine-guanosine-containing DNA oligodeoxynucleotides (ODNs) have been shown to be effective in reducing AD pathology in three AD animal models. To interrogate the involvement of TLR9 in AD, we measured the difference in methylation level of $\mathrm{CpG}$ probes in the promoter regions of TLR9 and demonstrated that hypermethylation of $\operatorname{cg} 11855705(\mathrm{p}=\mathbf{0 . 0 0 3})$ and hypomethylation of $\operatorname{cg} 08721301(\mathrm{p}=0.03)$, and $\operatorname{cg} 22484793(\mathrm{p}=\mathbf{0 . 0 3})$ in postmortem brain tissue from the superior temporal gyrus (STG) region in AD subjects comparing to cognitively normal control subjects. These results provided an independent line of evidence in the potential role of TLR9 in AD pathology.
\end{abstract}

Keywords: Epigenetic, Alzheimer's disease, Superior temporal gyrus, TLR9.

\section{Introduction}

Alzheimer's disease (AD) is a neurodegenerative disease and represents the most common cause of dementia. Neuritic plaques (NPs) consisting of amyloid $\beta$-protein $(A \beta)$ and neurofibrillary tangles (NFTs) comprised of hyper phosphorylated tau protein are two prominent neuropathological hallmarks of $\mathrm{AD}$. Neuroinflammation has been implicated in AD disease processes [1,2]. Fibrillar $A \beta$ deposits are closely associated with neuroinflammatory responses such as microglia activation and/or innate immune response. Toll-like receptors (TLRs) are a family of pattern recognition receptors in the innate immune system and have been implicated in clearance of $A \beta$ deposits in the brain $[3,4]$ and proposed to be therapeutic targets for $\mathrm{AD}[5]$. In particular, activation of microglia with the Toll-like receptor 9 (TLR9) ligand boosted ingestion of $\mathrm{A} \beta$ in vitro [3]. Stimulating the innate immune system via TLR9 with cytosine-guanosinecontaining DNA oligodeoxynucleotides (ODNs) in Tg2576 $\mathrm{AD}$ model transgenic mice reduced the cortical and vascular amyloid burden and $A \beta 42, A \beta 40$, and $A \beta$ oligomer levels [4]. Furthermore, this treatment also reduced both $A \beta$ and tau pathologies and levels of toxic oligomers and rescued cognitive deficits in 3xTg-AD mice, an animal model with both $A \beta$ and tau related pathology [6]. CpG ODNs treatment was also effective in Tg-SwDI, an animal model with abundant vascular amyloid association with low levels of parenchymal amyloid deposits, with effects in reducing cerebral amyloid angiopathy (CAA) pathology and negating memory deficit [7]. Transcription of TLR9 is up-regulated in control subjects upon $A \beta$ stimulation, while down regulated in AD patients [8]. However, there was also report of up-regulation of Tlr9 mRNAs in plaque material of aged APP23 transgenic mice compared to plaque-free tissue, while plaque-free tissue did not show an increased expression of any Tlr mRNAs compared to age-matched control mice [9]. TLR9 is also thought to play a role via sensing the methylation level of mitochondrial DNA released from damaged human cells and triggering the inflammatory cytokine cascade [10]. Furthermore, homozygote GG genotype from single nucleotide polymorphism rs187084 in TLR9 was significantly associated with a decreased LOAD risk after adjusting for age, gender, and ApoE $\varepsilon 4$ status $(\mathrm{P}=0.035)$ [11]. There was also a significant interaction between ApoE $\varepsilon 4$ and rs187084, with a stronger effect of rs187084 observed among e4 carriers $(\mathrm{P}<0.001$ in genotypic test and $\mathrm{p}=0.003$ in allelic test). The GG genotype of the TLR9 rs187084 polymorphism was associated with a higher TLR9 expression in peripheral blood monocytes.

Despite the genetic contribution in both familial and sporadic $\mathrm{AD}$, data from monozygotic twins discordant in AD development and onset [12-14] suggest that non-genetic factors play a key role. Epigenetic mechanism includes DNA methylation and covalent histone modification, which generate heritable changes in gene activity and expression without changing DNA sequence. Methylation level are often examined in three ways 1) global methylation level; 2) methylation level of $\mathrm{CpG}$ sites in candidate genes; and 3) epigenome wide association analysis (EWAS) with promising but sometimes inconsistent results. The epigenetic state is age, disease, disease stage, tissue, and cell type dependent which could contribute to the inconsistent results. Dysregulation of histone acetylation has been implicated in learning and memory, onset of age-associated memory impairment and the pathogenesis of neurodegenerative diseases. In an $\mathrm{AD}$ animal model, severe amyloid pathology correlated with a dysregulation of histone acetylation in the forebrain [15]. Learning and memory consolidation deficits and/or AD pathology reversal effects were demonstrated for histone deacetylases (HDAC) inhibitors such as phenylbutyrate $[16,17]$, sodium valproate [18], sodium butyrate [15,18-20], or vorinostat [18], suberoylanilide hydroxamic acid [19-21], trichostatin A [22] and environmental enrichment [23] in AD and/or brain-injured animal models. The recovery of learning/ memory function correlated with elevated histone acetylation 
[23], induced sprouting of dendrites, increased number of synapses [23], and increased expression of genes implicated in associative learning.

NP- and NFT-vulnerable brain regions such as superior temporal gyrus (STG) and middle temporal gyrus (MTG) regions of postmortem brains have been examined previously for global methylation level, at selected candidate locus, or at methylome wide [24-26]. In this study, we interrogated the difference in the STG methylation level of $\mathrm{CpG}$ probes in the putative regulatory regions of TLR9 to provide additional line of evidence in the involvement of TLR9 in AD.

\section{Material and Methods}

Samples from the STG region of postmortem brains from 91 Alzheimer's Disease patients and 60 healthy controls with normal cognition function were obtained from Banner Sun Health Research Institute under its brain donation program [27]. Genomic DNA and total RNA, including miRNA were simultaneous purified from the brain tissues samples using AllPrep DNA/RNA/miRNA Universal Kit (QIAGEN) following standard protocol. Genomic DNA was subject to genome wide methylation analysis using Infinium MethylationEPIC BeadChip (Illumina, San Diego, CA). All data generation were conducted by laboratory personnel blinded as to the clinical phenotype.

Quality control of the epigenetic data was performed using R package ChAMP [28]. Probes that did not perform well (with detection $p$-value $\geq 0.01$ in one or more samples, or with bead count $>3$ in at least $5 \%$ of samples), probes with known SNP sites, non-cg probes, probes align to multiple locations on the genome, as well as probes located on the sex chromosomes were filtered out. The final dataset contained 753,038 probes and 151 samples.

The methylation levels were then normalized using Dasen method from $\mathrm{R}$ package WaterMelon [29]. The proportion of NeuN+ cells (primarily neurons) was estimated using the estimate Cell Counts function in $\mathrm{R}$ package minfi [30]. We used $\mathrm{R}$ package sva [31] to detect hidden batch effect in the samples and included the top five surrogate variables generated in addition to sex and age as covariates in the linear regression model to identify differentially methylated probes between AD patients and cognitively normal controls. For this study, only probes from TLR9 region was examined and no multiple testing correction was made.

\section{Results}

Post-mortem samples used in the epigenetic analysis were described in Table 1 with the age of AD patients slightly older than the age of cognitively normal patients. TLR9 is transcribed from the reverse complement strand of the human genome, there are nine $\mathrm{CpG}$ probes from Infinium Methylation EPIC BeadChip within 1500 base pair upstream of transcript start site (TSS) of TLR9 (e.g. upstream of the transcriptional start). We observed a hypermethylation of CpG site $\operatorname{cg} 11855705(\mathrm{p}=0.003)$ and hypomethylation of $\operatorname{cg} 08721301(\mathrm{p}=0.03)$, and $\operatorname{cg} 22484793$ $(\mathrm{p}=0.03)$ (Figure 1 and Table 2$)$ in the putative promoter region of TLR9 in AD subjects in the superior temporal gyrus (STG) region of postmortem brains from $\mathrm{AD}$ patients comparing to cognitively normal patients.

\section{Discussion}

Innate immunity seems to play opposing roles during the $\mathrm{AD}$ progression, e.g. activated microglia and reactive astrocytes exert neuroprotection mediated through $A \beta$ phagocytosis in the early stage, whereas they fail in $A \beta$ clearance and therefore exert detrimental effects including neuroinflammation and neurodegeneration as the disease progresses. This may be the reason behind opposing directionality in expression level observed in various experimental settings. In this study, the net methylation consequence of the three $\mathrm{CpG}$ sites is unknown as hypermethylation of $\operatorname{cg} 11855705$ presumably would result in a predicted lower expression of TLR9 transcript level and consistent with the peripheral results reported by Fiala et al. and Wang et al., while hypomethylation of $\operatorname{cg} 08721301$ and cg22484793 presumably would result in a predicted higher

Table 1. Samples used in the epigenetic analysis.

\begin{tabular}{|c|c|c|c|}
\hline Variables & Alzheimer's Disease $(n=91)$ & Cognitively Normal $(n=60)$ & P-value \\
\hline Age (years, mean \pm SD) & $83.93 \pm 8.84$ & $80.65 \pm 6.88$ & $0.005^{*}$ \\
\hline Gender (male, \%) & $44(48.4 \%)$ & $37(61.7 \%)$ & $0.13^{* *}$ \\
\hline
\end{tabular}

A

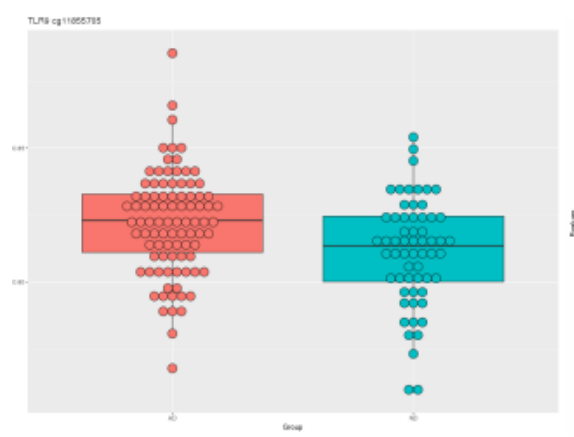

B

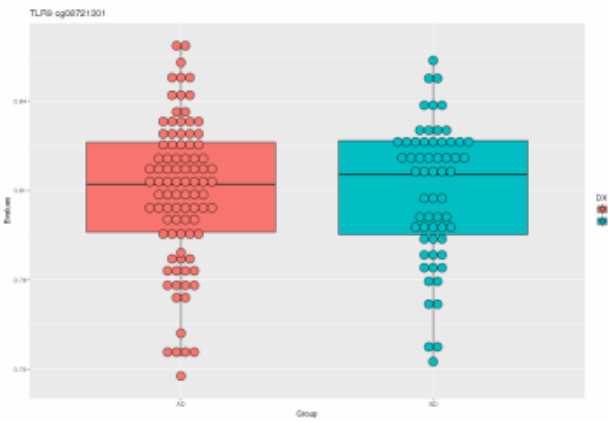

C

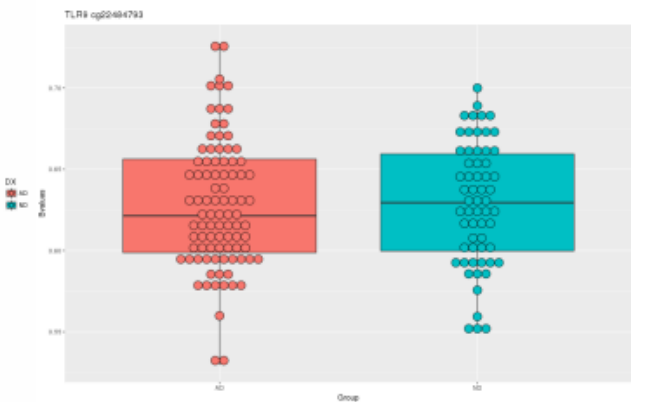

Figure 1. Differential methylation positions (DMP) upstream of TLR9 in AD vs. cognitively normal subjects (A) cg11855705 (B) cg08721301 (C) cg22484793. 
Table 2. DMP in AD subjects vs. controls.

\begin{tabular}{|c|c|c|c|c|c|c|c|c|c|c|c|}
\hline chr & pos & strand & Name & $\begin{array}{l}\text { Relation_to_ } \\
\text { Island }\end{array}$ & $\begin{array}{c}\text { UCSC_RefGene_ } \\
\text { Group }\end{array}$ & $\log F C$ & AveExpr & $\mathbf{t}$ & $p$ value & $\begin{array}{l}\text { adj } P \\
\text { Val }\end{array}$ & B \\
\hline chr3 & 52260373 & + & cg11855705 & Open Sea & TSS200 & -0.12 & 2.17 & -3.07 & 0 & 0.21 & -1.63 \\
\hline chr3 & 52260227 & + & cg08721301 & Open Sea & TSS200 & 0.09 & 2.1 & 2.16 & 0.03 & 0.45 & -3.67 \\
\hline chr3 & 52261325 & - & cg22484793 & Open Sea & TSS1500 & 0.11 & 0.76 & 2.16 & 0.03 & 0.45 & -3.67 \\
\hline chr3 & 52260349 & - & cg01395047 & Open Sea & TSS200 & 0.04 & 0.85 & 1.52 & 0.13 & 0.64 & -4.72 \\
\hline chr3 & 52260965 & - & cg05778154 & Open Sea & TSS1500 & -0.05 & 2.37 & -1.37 & 0.17 & 0.69 & -4.91 \\
\hline chr3 & 52260671 & + & cg18452449 & Open Sea & TSS1500 & 0.03 & 1.64 & 0.97 & 0.33 & 0.8 & -5.34 \\
\hline chr3 & 52260280 & - & cg17716965 & Open Sea & TSS200 & -0.01 & 1.38 & -0.48 & 0.63 & 0.92 & -5.66 \\
\hline chr3 & 52260432 & - & cg16302310 & Open Sea & TSS1500 & -0.01 & 2.26 & -0.2 & 0.84 & 0.97 & -5.75 \\
\hline chr3 & 52261446 & - & cg26207095 & Open Sea & TSS1500 & 0 & 1.76 & 0.08 & 0.93 & 0.99 & -5.76 \\
\hline
\end{tabular}

expression of TLR9 transcript level and consistent with the Frank et al. where Tlr9 mRNAs in plaque material of aged APP23 transgenic mice was upregulated compared to plaquefree tissue and control tissues.

\section{Conclusion}

Overall, the study provides an independent line of evidence from epigenetic mechanism in the potential role of TLR9 in AD in a brain region vulnerable to $\mathrm{AD}$ pathology. Further replication evidence will be needed.

\section{Acknowledgement}

We thank the patients for participating in the brain donor program and the researchers at Banner Sun Research Institutes for making the samples available to research communities. We also thank the staff at Cancer Genetics, Inc. for extracting nucleotide from the samples, quality control, and plating the samples for assay, and the staff at Illumina for generating the epigenetic assay data.

\section{References}

1. Bagyinszky E, Giau VV, Shim K, et al. Role of inflammatory molecules in the Alzheimer's disease progression and diagnosis. J Neurol Sci. 2017;376:242-54.

2. Le Page A, Dupuis G, Frost EH, et al. Role of the peripheral innate immune system in the development of Alzheimer's disease. Exp Gerontol. 2017.

3. Tahara K, Kim HD, Jin JJ, et al. Role of toll-like receptor signalling in $A \beta$ uptake and clearance. Brain. 2006;129:3006-19.

4. Scholtzova H, Kascsak RJ, Bates KA, et al. Induction of toll-like receptor 9 signaling as a method for ameliorating Alzheimer's disease-related pathology. J Neurosci.2009;29:1846-54.

5. Gambuzza EM, Sofo V, Salmeri MF, et al. Toll-like receptors in Alzheimer's disease: A therapeutic perspective. CNS Neurol Disord Drug Targets. 2014;13:1542-58.

6. Scholtzova H, Chianchiano P, Pan J, et al. Alzheimer's disease related pathology is reduced by Toll-like receptor 9 stimulation. Acta Neuropathol Commun. 2014;2:101.

7. Scholtzova $\mathrm{H}$, Do $\mathrm{E}$, Dhakal $\mathrm{S}$, et al. Innate immunity stimulation via toll-like receptor 9 ameliorates vascular amyloid pathology in Tg-SwDI mice with associated cognitive benefits. J Neurosci. 2017;37:936-59.
8. Fiala M, Liu PT, Espinosa-Jeffrey A, et al. Innate immunity and transcription of MGAT-III and Tolllike receptors in Alzheimer's disease patients are improved by bisdemethoxycurcumin. P Natl Acad Sci. 2007; 104:12849-54.

9. Frank S, Copanaki E, Burbach GJ, et al. Differential regulation of toll-like receptor mRNAs in amyloid plaqueassociated brain tissue of aged APP23 transgenic mice. Neurosci Lett. 2009;453:41-4.

10. Clark IA, Vissel B. Treatment implications of the altered cytokine-insulin axis in neurodegenerative disease. Biochem Pharmacol. 2013;86:862-71.

11. Wang YL, Tan MS, Yu JT, et al. Toll-like receptor 9 promoter polymorphism is associated with decreased risk of Alzheimer's disease in Han Chinese. J Neuroinflammation. 2013;10:886.

12. Plomin R, Owen MJ, McGuffin P. The genetic basis of complex human behaviors. Science. 1994;264:1733-9.

13. Gatz M, Pedersen NL, Berg S, et al. Heritability for Alzheimer's disease: The study of dementia in Swedish twins. J Gerontol A Biol Sci Med Sci. 1997;52:M117-25.

14. Mastroeni D, McKee A, Grover A, et al. Epigenetic differences in cortical neurons from a pair of monozygotic twins discordant for Alzheimer's disease. PloS one. 2009; 4:e6617.

15. Govindarajan N, Agis-Balboa RC, Walter J, et al. Sodium butyrate improves memory function in an Alzheimer's disease mouse model when administered at an advanced stage of disease progression. J Alzheimers Dis. 2011;26:187-97.

16. Ricobaraza A, Cuadrado-Tejedor M, Pérez-Mediavilla A, et al. Phenylbutyrate ameliorates cognitive deficit and reduces tau pathology in an Alzheimer's disease mouse model. Neuropsychopharmacology. 2009;34:1721-32.

17. Ricobaraza A, Cuadrado-Tejedor M, Marco S, et al. Phenylbutyrate rescues dendritic spine loss associated with memory deficits in a mouse model of Alzheimer disease. Hippocampus. 2012;22:1040-50.

18. Kilgore M, Miller CA, Fass DM, et al. Inhibitors of class 1 histone deacetylases reverse contextual memory deficits in a mouse model of Alzheimer's disease. Neuropsychopharmacology. 2010;35:870. 
19. Fontán-Lozano Á, Romero-Granados R, Troncoso $\mathrm{J}$, et al. Histone deacetylase inhibitors improve learning consolidation in young and in KA-inducedneurodegeneration and SAMP-8-mutant mice. Mol Cell Neurosci. 2008;39:193-201.

20. Dash PK, Orsi SA, Moore AN. Histone deactylase inhibition combined with behavioral therapy enhances learning and memory following traumatic brain injury. Neuroscience. 2009;163:1-8.

21. Peleg S, Sananbenesi F, Zovoilis A, et al. Altered histone acetylation is associated with age-dependent memory impairment in mice. Science. 2010;328:753-6.

22. Francis YI, Fà M, Ashraf $H$, et al. Dysregulation of histone acetylation in the APP/PS1 mouse model of Alzheimer's disease. J Alzheimers Dis. 2009;18:131-9.

23. Fischer A, Sananbenesi F, Wang X, et al. Recovery of learning and memory is associated with chromatin remodelling. Nature. 2007;447:178-82.

24. Ellison EM, Abner EL, Lovell MA. Multiregional analysis of global 5-methylcytosine and 5-hydroxymethylcytosine throughout the progression of Alzheimer's disease. J Neurochem. 2017;140:383-94.

25. Sontag E, Hladik C, Montgomery L, et al. Downregulation of protein phosphatase 2A carboxyl methylation and methyltransferase may contribute to Alzheimer disease pathogenesis. J Neuropathol Exp Neurol. 2004;63:1080-91.

26. Lunnon K, Smith R, Hannon E, et al. Methylomic profiling implicates cortical deregulation of ANK1 in Alzheimer's disease. Nat Neurosci. 2014;17:1164-70.

27. Beach TG, Sue LI, Walker DG, et al. The Sun Health Research Institute Brain Donation Program: Description and experience, 1987-2007. Cell Tissue Bank. 2008;9:229-45.

28. Morris TJ, Butcher LM, Feber A, et al. ChAMP: $450 \mathrm{k}$ chip analysis methylation pipeline. Bioinformatics. 2013;30:428-30.

29. Pidsley R, Wong CC, Volta M, et al. A data-driven approach to preprocessing Illumina $450 \mathrm{~K}$ methylation array data. BMC Genomics. 2013;14:293.

30. Aryee MJ, Jaffe AE, Corrada-Bravo H, et al. Minfi: A flexible and comprehensive Bioconductor package for the analysis of Infinium DNA methylation microarrays. Bioinformatics. 2014;30:1363-9.

31. Leek JT, Johnson WE, Parker HS, et al. The SVA package for removing batch effects and other unwanted variation in high-throughput experiments. Bioinformatics. 2012;28:882-3.

\section{*Correspondence to:}

Qingqin S. Li

Neuroscience Therapeutic Area

Janssen Research and Development, LLC

1800 American Blvd

Pennington, New Jersey

USA

Tel: 6097304576

E-mail: qli2@its.jnj.com 